\title{
Paradoxical side effect in treatment with antipsychotics for aggression/agitation and psychosis in dementia: case-series
}

\author{
Tudor Florea, Matei Palimariciuc, Vasile Chiriță, Roxana Chiriță
}

\begin{abstract}
Tudor Florea - M.D., PhD. Student, assistant professor Grigore T. Popa University of Medicine and Pharmacy, Iași, Psychiatrist, "Sf. Sava" Hospital, Iași, Romania

Matei Palimariciuc - M.D., PhD Student, assistant professor Grigore T. Popa University of Medicine and Pharmacy, Iași; junior psychiatrist, Socola Institute of Psychiatry, Iași

Vasile Chiriță - M.D., PhD, Professor of Psychiatry, Honorary Member of the Romanian Academy of Medical Science

Roxana Chiriță - M.D., PhD, Professor of Psychiatry Grigore T Popa University of Medicine and Pharmacy, Iași, Senior psychiatrist
\end{abstract}

\begin{abstract}
Antipsychotic treatment is widely used for treating aggression/agitation and psychosis in the elderly population. The doses used are lower, administering medication is done over a prolonged time period, tests done and clinical observation is more frequent compared to the non-geriatric population. The objective of this case-series was to present a particularity encountered in the use of antipsychotics. Three patients suffered from increased aggression and agitation during treatment with antipsychotics. After medication administration was ceased the symptomatology remitted. Tests used for this study included Mini-Mental State Exam (MMSE), Neuropsychiatric Inventory Nursing Home Version (NPI-NH). We found that the elevation of the NPI-NH score was significant with increased dosage, thus after reaching the maximum allowed doses for patients with dementia, the decision was made to stop the antipsychotic and change to another agent (antipsychotic or anticonvulsant). These results suggest that more studies should focus on fast management of the antipsychotics' side effects, and the dynamics between symptom development and treatment decisions. The delay between the start of medication and the decision to switch could have a negative impact for the patients. Future treatment algorithms should take into consideration the possibility of worsening the symptoms and more in-depth studies should be conducted to widen the picture of antipsychotic treatment in the geriatric population, especially for patients with severe dementia.
\end{abstract}


KEY WORDS:

\section{Antipsychotics, dementia, palliative care, paradoxical side effect.}

\section{INTRODUCTION}

According to the World Health Organization around 50 million people have dementia, with nearly 10 million new cases every year and a projection of 82 million by 2030 and 152 million by 2050. (1) Population aging is a main contributing factor to the emergence of the dementia epidemic, by 2050 it is presumed that $22 \%$ of the world population will be over 60 years. (2) Dementia is a clinical syndrome caused by progressive neurodegeneration and is characterized by deterioration in cognitive ability. Other important non-cognitive manifestations are represented by a group of behavioral and psychological symptoms of dementia (BPSD), up to $80 \%$ of patients develop at some stage of dementia a form of BPSD. (3) BPSD is associated with impaired quality of life, and include anxiety, depression, hallucinations and delusions, agitation, aggression, hypersexuality, wandering, sleeping and eating problems. The management of BPSD represents a challenge for the geriatric psychiatry units, no medication algorithm can be applicable to every patient, and thus the physician's impact in decision making remains of primary importance. (4)

The association of dementia and the presence of psychosis is a main factor for the rapid cognitive decline, the main goal in treating these patients being the preservation of the cognitive capacity through different measures. Antipsychotic medication is frequently used in treating agitation associated with delirium, psychosis, aggression or anger. (5) The profile of such drugs leads to regulation of use for geriatric patients, limiting the negative impact that these drugs have on the elder population. Most of the clinical trials conducted in later years provided evidence for the use of atypical antipsychotics (i.e. risperidone) with safety advantages and higher effectiveness over other classes of medication. The clinical trials for the sideeffect profile of atypical antipsychotics bring evidence for less sedation, lesser postural instability, movement disorders and cardiovascular risk. (6). For other classes of psychotropic medication such as antidepressants, mood stabilizers, benzodiazepines there is little evidence for a positive risk-benefit ratio in long-term treatment. (7)

The use of atypical antipsychotics should be used with deliberate consideration, and the prescribed doses need to be adjusted to maximize efficacy and minimize the side effect profile. Clinical improvement should be expected within 12 weeks, the absence of a positive effect should orient toward revisiting the BPSD assessment, changing the antipsychotic or other behavioral and environmental interventions. There is no evidence for a positive outcome in switching from an atypical antipsychotic to nonantipsychotic drugs in such cases, even though the literature for such algorithms is scarce. (8)

The expert consensus guideline for dementia treatment and its behavioral disturbances indicate Risperidone as first line treatment for agitation/aggression in dementia. Initial doses range between $0.25-0.5 \mathrm{mg} /$ day with the possibility, after assessment, of raising the doses towards a maximum of $2 \mathrm{mg} /$ day. With lower efficacy, the use of Quetiapine and Aripiprazole as second-line is advised when Risperidone failed to reduce the symptoms, evidence also existing for Carbamazepine, Gabapentin or a combination of two of the 
r

above-mentioned substances. (4) In Romania, therapy protocols supported by the CNAS (Casa Naţională de Asigurări de Sănătate) for treatment of agitation/aggression in dementia recommends Risperidone for a maximum of 6 weeks as the sole option in such cases. The use of other drugs does not have at the moment financial support or indication from the healthcare system. (10)

\section{METHODS}

\section{Setting}

A study was conducted in 3 patients with dementia who were in palliative care between May 2019 and February 2020. All three patients had been taking anti-dementia agents for more than 6 months (Memantine), no change in cardiovascular or other type of medication was recorded prior or during the study. First assessment included diagnosis of dementia according to ICD-10 criteria, sex, age, weight, history of alcohol and tobacco use, medical and surgical history, full physical examination, laboratory analysis and electrocardiogram. For our group of patients, antipsychotic medication was initiated according to the expert consensus guidelines for dementia treatment and BPSD, in accordance with the national legislation and guidelines. Risperidone was the medication of choice for all three cases, dose adjustments were conducted according to the expert consensus guidelines. $(4,11)$ In these cases, follow-up evaluation was conducted, clinical assessment occurred periodically, NPI-NH testing occurred at week 1, when the medication was discontinued and after two weeks with the new medication. Patients received specific care according to the protocols, consisting in hydration, psychosocial support and focused interventions.

\section{Test batteries}

For the assessment of dementia and for screening purposes Mini-Mental State
Examination (MMSE) was used. MMSE is a cognitive test that is commonly utilized in the diagnostic and evaluation of dementia. (12)

For the assessment of psychosis, agitation and aggressive behavior Neuropsychiatric Inventory Nursing Home Version (NPI-NH) scale was used at two distinctive moments, at the moment of initiation of the antipsychotic treatment and after the maximum dose was reached. This instrument assesses the neuropsychiatric symptoms and psychopathology of patients with dementia. Professional caregivers administered the NPI$\mathrm{NH}$ scale. The test includes ten behavioral subsections and two types of neurovegetative changes. Every subsection has one frequency and one severity aspect built into the questionnaire. (13)

Data was collected from the medical chart of each patient. The protocols for treating all the patients that meet the criteria for BPSD were conducted in a similar manner, variations are made on a case by case basis, according to the clinical estimation and patient's need.

\section{RESULTS}

Three patients (all males) were enrolled in the study. All three patients developed a form of BPSD with aggression and agitation for which antipsychotic medication was started. All three cases have in common the development of a paradoxical reaction to the treatment, the intensity of aggression and agitation increased with higher doses of antipsychotic. All the patients were in treatment with Memantine and had no changes in the somatic treatment schemes for more than 6 months prior to the start point of the study. The data is summarized in Table 1.

The patients were tested for infection, renal failure and other possible somatic diseases; temperature, blood pressure, cardiac 
s frequency and other vital signs were The third NPI-NH control test, done after monitored. There was no modification in the medication was switched to another agent, described parameters compared to the average showed an improvement for all three cases. of 6 months prior to the episode.

\begin{tabular}{l|l|l|l|l}
\hline \multicolumn{5}{c}{ Table 1. Patients before medication } \\
\hline Variable & 1 & 2 & 3 & Avg \\
Sex & $\mathrm{M}$ & $\mathrm{M}$ & $\mathrm{M}$ & \\
Age (yrs.) & 82 & 69 & 69 & 73.3 \\
Weight (kg) & 74 & 78 & 72 & 74.6 \\
Alcohol history & No & No & Yes & 7 \\
MMSE & 10 & 5 & 6 & 2.6 \\
DoD (yrs.) & 2 & 4.5 & 3.5 & \\
Stroke history & Yes & No & Yes & \\
Aggression history & Yes & No & No & \\
\hline Values are presented as data only or mean. M-male \\
Mini-Mental State Examination (MMSE) \\
\hline
\end{tabular}

\section{Case 1}

The first patient was a 82 years old male patient with personal history of stroke and cardiovascular disease. The patient had a history of aggression towards his children, according to the family, this type of behavior diminished with time. The patient had a college degree and worked as a composer. There was no history of alcohol drinking or use of other substances. The onset of dementia was first slow and after fast paced, the diagnostic was established as dementia mixed type. The current development of the psychosis and aggression was sudden, the patient described visual and auditory hallucinations and a form of delusion concerning kidnapping. The patient was assessed with NPI-NH and antipsychotic medication, initial dose $0.5 \mathrm{mg}$, was initiated. After a small improvement, the patient relapsed and the doses were increased, over a period of 24 days, the dose of $2 \mathrm{mg} /$ day was reached. Even though the first period had some symptom fluctuations, with the increase in dosage, the patient developed increased impulsivity, aggressive behavior and delusions. Because of these manifestations, the decision was made to switch to another antipsychotic. In order to facilitate the transition to the other medication without side effects, overlapping was avoided. After dose reduction, the above-mentioned symptoms diminished in intensity, without disappearing.

\section{Case 2}

The second patient was a 69 years old male, having no history of stroke or cardiovascular disease. There was no history of aggression or alcohol drinking. The patient met the diagnostic criteria for dementia in Alzheimer's disease. The patient worked as engineer and no toxic environment was reported. The evolution of agitation was slow, the patient presented in the beginning the inability to sit for more than 5 minutes. With time, aggression towards the medical staff grew in intensity, so that antipsychotic medication was initiated. A similar protocol was used as for case 1 . Because symptoms had a lower intensity, the doses were raised over a longer period and after 32 days, the maximum dose was reached. With the increase in dose, the 
Bulletin of Integrative Psychiatry $\bigcirc$ New Series OJune 2020 ○ Year XXVI ONo. 2 (85)/123

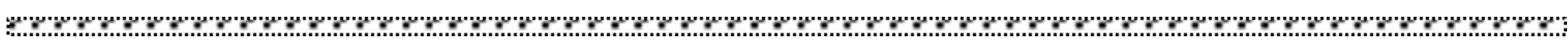

patient developed gradually more agitation. After the medication was ceased, the symptoms diminished in intensity. After switching a residual symptomatology remained.

\section{Case 3}

The third patient was male, 69 years old, with history of stroke and cardiovascular disease. The patient worked as a professional football player, had no history of aggression, but suffered from alcohol dependence. He was abstinent for more than 10 years. The symptoms debuted with agitation, sleeping disorders, restlessness, and evolved towards physical aggression. Antipsychotic medication was initiated, and a dose of 2 $\mathrm{mg} /$ day was reached in 24 days. The patient managed to break the furniture in his room and made holes in the wall with different objects Because the patient became more aggressive, the decision was made to switch to another medication.

For all the patients the NPI-NH was performed on the first day upon treatment initiation and a week after the maximum dose was reached. The results can be found in Table 2.

\begin{tabular}{|c|c|c|c|c|}
\hline \multicolumn{5}{|c|}{ Table 2. NPI-NH Score } \\
\hline Variable & Pati & & & \\
\hline & 1 & 2 & 3 & Avg \\
\hline NPI-NH & & & & \\
\hline Initial & 31 & 22 & 30 & 27.6 \\
\hline Latest & 50 & 53 & 64 & 55.6 \\
\hline NPI-NH (Aggression and Aberrant Motor & & & & \\
\hline Behavior & & & & \\
\hline Initial & 14 & 8 & 10 & 10.6 \\
\hline Latest & 24 & 23 & 28 & 25 \\
\hline
\end{tabular}

The evolution of each patient was documented thoroughly by the medical personal on a daily basis thus, we managed to extract the information and interpret for agitation/aggression and irritability. The data was used to create a graphic with exponential tendencies (Figure 1). The evolution of the symptomatology correlated with the clinical observation. 


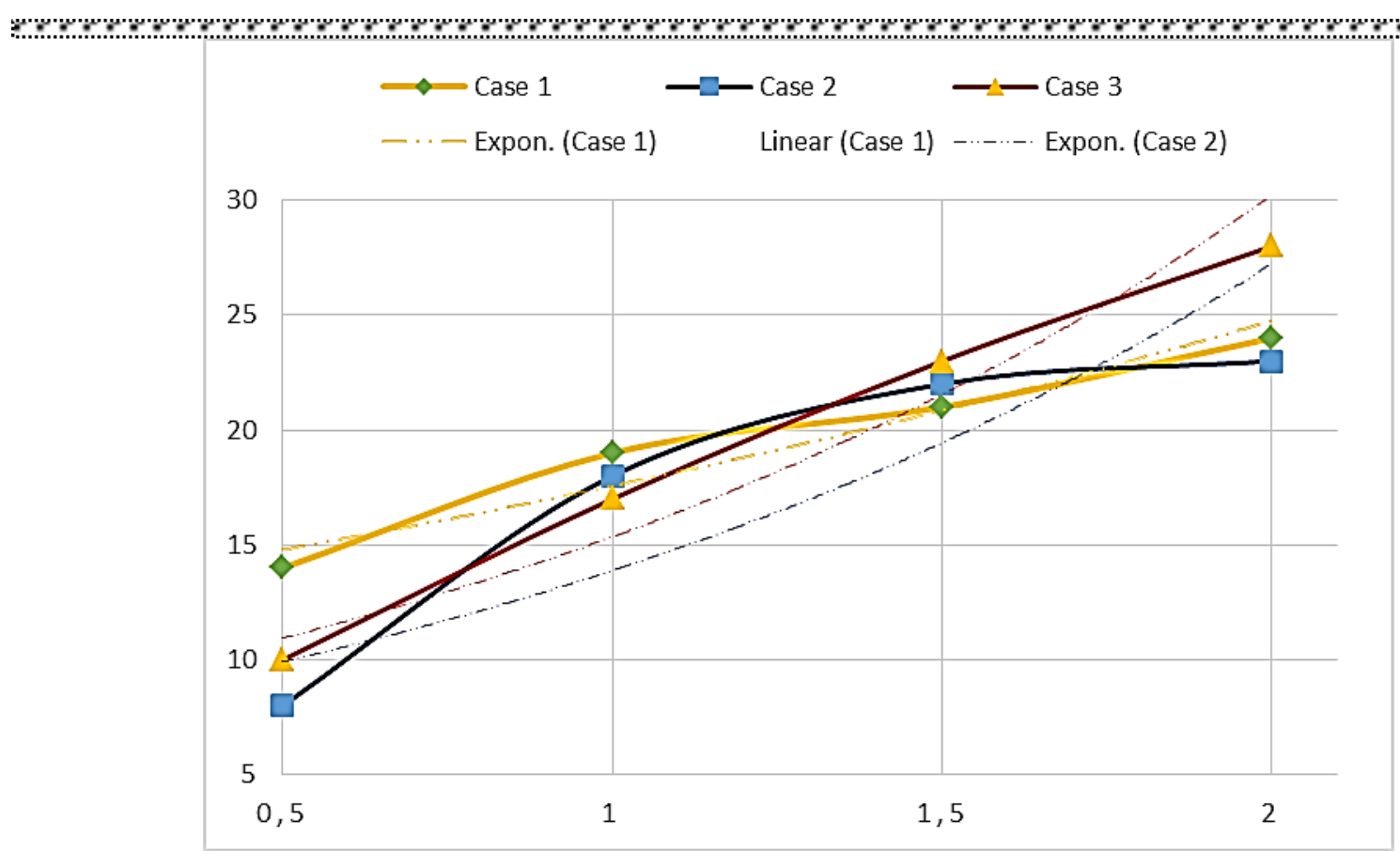

Figure 1. NPI-NH Score for Aggression and Aberrant Motor Behavior

On the Y-axis the sum of scores for aggression and Aberant Motor Behavior

On the $\mathrm{X}$-axis, the total dose of antipsychotic reached

With the dotted line the exponential function was graphed for each case

\section{DISCUSSION}

In medical geriatric practice the antipsychotic medication is commonly used, the extend worldwide is still unknown.(14) For our general practice, the results using Risperidone for aggression/agitation and psychosis are positive and very well documented. Moreover, the short-term side effects are few, the antipsychotic treatment is a protective factor in our analysis. (15) Other studies conducted show a clear improvement for symptoms such as anger, aggression and paranoid ideas, without improving conclusively the quality of life. $(16,17)$

We have mentioned these three cases for their particularities during treatment for BPSD. Our clinical observations are consistent with the main data regarding the use of antipsychotics in the elderly population, and our best results are with Risperidone. Nevertheless, the observation of the paradoxical side effects had no correlation in any compelling guidelines or the extended literature for a fast reaction to this type of development. The treatment algorithm consensus advises waiting and dose increase.

Even though the disorder developed paradoxically, the initial low intensity of the symptoms was concluded to be derived from the low doses used. The decision to maintain the treatment with the chosen antipsychotic was also influenced by the lack of alternative from the Romanian Health Care System's part. The use of off-label medication (other antipsychotics or drugs) for aggression / agitation in dementia has no financial support, thus a long-term treatment with other agents would be a burden for the patient and his family. (18)

Although the side-effects of antipsychotics are clearly stated, their evolution in the elderly patient and the future patient management is inconclusive and may result in flawed decision-making. Studying these cases, we found ourselves in the position of 
not knowing how symptoms will evolve, if an increased dose would solve the initial manifestations, and what would be the best alternative, another antipsychotic or other class agents such as anticonvulsive medication.
We haven't encountered other typical side effects such as extrapyramidal symptoms or increased fall risk, dizziness, cardiovascular events or other neurologic symptoms. Complications as mentioned have an impact on care needs, mortality and overall quality of life. (18)

\section{CONCLUSIONS}

Treatment of aggression/agitation and psychosis remains a primary focus for the geriatric care. New treatment algorithms are tested and published and an overall consensus is still to be desired in developing such clear protocols for antipsychotic usage in elderly patients.

Case-series reports present a major importance for future studies looking at reducing the frequency of side effects. It also has a fundamental role for practitioners to improve their case by case management with identification of key symptoms and evolutions. In this manner, a fast, fact-based decision could be made and prevent future complications.

There is a strong need in Romania for alternative medication for aggression/agitation in dementia that has support from the NHS in accordance with international standard practice.

Further examinations are required to determine the best solution then facing these types of side effects and guidelines should primarily focus on the symptoms' development over time and its correlation with antipsychotic doses.

\section{ACKNOWLEDGEMENTS AND DISCLOSURES}

The authors declare that they have no potential conflicts of interest to disclose.

\section{REFERENCES}

1. World Health Organisation: Dementia fact sheet. WHO, 2019 https://www.who.int/news-room/factsheets/detail/dementia?

2. Prince, M., Bryce, R., Albanese, E., Wimo, A., Ribeiro, W., \& Ferri, C. P. (2013). The global prevalence of dementia: a systematic review and metaanalysis. Alzheimer's \& dementia, 9(1), 63-75.

3. Lyketsos, C. G., Lopez, O., Jones, B., Fitzpatrick, A. L., Breitner, J., \& DeKosky, S. (2002). Prevalence of neuropsychiatric symptoms in dementia and mild cognitive impairment: results from the cardiovascular health study. Jama, 288(12), 1475-1483.

4. Davies, S. J., Burhan, A. M., Kim, D., Gerretsen, P., Graff-Guerrero, A., Woo, V. L.\& Rajji, T. K. (2018). Sequential drug treatment algorithm for agitation and aggression in Alzheimer's and mixed dementia. Journal of Psychopharmacology, 32(5), 509-523.

5. Alexopoulos, G. S., Silver, J. M., Kahn, D. A., Frances, A., \& Carpenter, D. (1998). Treatment of agitation in older persons with dementia: The Expert Consensus Guideline Series. Postgraduate Medicine, A Special Report.)

6. Brodaty, H., Ames, D., Snowdon, J., Woodward, M., Kirwan, J., Clarnette, R., ... \& Grossman, F. (2003). A randomized placebo-controlled trial of risperidone for the treatment of aggression, agitation, and psychosis of dementia. Journal of Clinical Psychiatry, 64(2), 134-143.

7. Sink, K. M., Holden, K. F., \& Yaffe, K. (2005). Pharmacological treatment of neuropsychiatric symptoms of dementia: a review of the evidence. Jama, 293(5), 596-608.

8. Schneider, L. S., Dagerman, K., \& Insel, P. S. (2006). Efficacy and adverse effects of atypical antipsychotics for dementia: meta-analysis of randomized, placebo-controlled trials. The American Journal of Geriatric Psychiatry, 14(3), 191-210.

9. Hosie A, Davidson PM, Agar M, Sanderson CR, Phillips J. Delirium prevalence, incidence, and implications for screening in specialist palliative care inpatient settings: a systematic review. England; 2013 Jun. 
10. http://www.casan.ro/casar//theme/cnas/js/ckeditor/filemanager/userfiles/ProtocoaleTerapeuticeANEXELE_Nr1-

49doc.pdf

11. McDermott, C. L., \& Gruenewald, D. A. (2019). Pharmacologic Management of Agitation in Patients with Dementia. Current geriatrics reports, 8(1), 1-11.

12. Creavin ST, Wisniewski S, Noel-Storr AH, et al. Mini-Mental State Examination (MMSE) for the detection of dementia in clinically unevaluated people aged 65 and over in community and primary care populations. England; 2016 Jan 13.

13. Zuidema SU, Buursema AL, Gerritsen, Maarten G J M, et al. Assessing neuropsychiatric symptoms in nursing home patients with dementia: reliability and Reliable Change Index of the Neuropsychiatric Inventory and the CohenMansfield Agitation Inventory. Int J Geriatr Psychiatry. 2011;26(2):127-134.

14. Kamble P, Chen H, Sherer JT, Aparasu RR. Use of antipsychotics among elderly nursing home residents with dementia in the US: an analysis of National Survey Data. Drugs Aging. 2009;26(6):483-492.

15. Florea T, Palimariciuc M, Chirita R. Antipsychotic treatment in patients with dementia receiving palliative care: efficiency, fall risk and cognitive impact. Bulletin of Integrative Psychiatry:2019;83(4):79-87.

16. Sultzer DL, Davis SM, Tariot PN, et al. Clinical symptom responses to atypical antipsychotic medications in Alzheimer's disease: phase 1 outcomes from the CATIE-AD effectiveness trial. Am J Psychiatry.2008;165(7):844-854.

17. Schneider LS, Dagerman K, Insel PS. Efficacy and adverse effects of atypical antipsychotics for dementia: metaanalysis of randomized, placebo-controlled trials. Am J Geriatr Psychiatry. 2006;14(3):191-210.Creavin ST, Wisniewski S, Noel-Storr AH, et al. Mini-Mental State Examination (MMSE) for the detection of dementia in clinically unevaluated people aged 65 and over in community and primary care populations. England; 2016 Jan 13.

18. CNAS - site 2020 : http://www.cnas.ro/page/lista-medicamentelor-2019.html

Burns E, Kakara R. Deaths from Falls Among Persons Aged /=65 Years - United States, 2007-2016. MMWR Morb Mortal Wkly Rep. 2018;67(18):509-514.

\section{Correspondence:}

Matei Palimariciuc,

PhD Student, assistant professor Grigore T. Popa University of Medicine and Pharmacy, Iași; junior psychiatrist, matei.palimariciuc@umfiasi.ro

Submission: 28 mar 2020

Acceptance: 19 may 2020 